\title{
CYCLE TIME OPTIMIZED PATH PLANNING FOR INDUSTRIAL ROBOT USING ROBOMASTER
}

\section{SUPRIYA SAHU \& B. B CHOUDHURY}

Department of Mechanical Engineering, I.G.I.T Sarang, Odisha, India

\begin{abstract}
The main aim of the paper is to demonstrate the optimal cycle time taken by a 6 axis industrial robot, by following the shortest path. Planning for path is important because, it allows knowing about the path traversal of robot from starting point and reaching the end point with shortest possible time in $3 D$ workspace. The approach of this work is based on the use of robomaster software, for solving the path planning problems for industrial robot arms, that are used in industries and for any dynamic environments. In this analysis, an attempt has been taken to plan the path followed by the robot, by considering different sort options with different starting points. The analysis of different paths with cycle time and path length are done and from that, one having the shortest cycle time and minimum path length is achieved, which can be considered for further machining works.
\end{abstract}

KEYWORDS: Cycle Time, Industrial Robot, Path Planning, Robomaster and Workspace

Received: Jul 29, 2017; Accepted: Aug 18, 2017; Published: Aug 29, 2017; Paper Id.: IJMPERDOCT201710

\section{INTRODUCTION}

The performance of industrial robot is more important by considering their movement with high speeds, short time and with large accelerations. The use of robot in production sector depends upon the efficiency with which it performs a given task with shortest possible time, which in turn predicts the production rates. The highest priority given to the optimisation criterion is, the robot cycle times considering the limits of robot such as, maximum motor speeds and robot arm design etc. Also energy consumptions of the motors can be used as additional lower priority criteria, which are not considered for our work. Therefore, a good specification of robot is essential to get economically sound performance.

Carlason et al. [1] worked on dimensional quality and cycle time, based on search algorithm and proposed a novel method for optimization. Ulrich et al. [2] improved the flexibility of inspection system, based on robots by generating an automated path planning algorithm, which gives collision free path and optimised the time. Abele et al. [3] generated the time optimal tool paths, for a robot based deburring process of internal contours, by Matlab interface to find the shortest path by solving the travelling salesman problem. Gigras et al. [4] presented a new ant colony based approach, which is helpful in solving path planning problem for autonomous robotic application. Willigenburg et al. [5] used kinematic path planner, to find the minimum time taken for the path planning and proposed a new computational method. Cristina Castejon et al. [6] proposed the design criteria using computationally efficient objective function and investigated the main characteristic of service tasks. Saravanan et al. [7] proposed a method to find out optimal motion of a robot arm, in the presence of a fixed as well as oscillating obstacles, in the path of robot structure. Rahman et al. [8] proposed a framework for determining workspace of industrial robot, for providing a fast and easy configuration approach, for safe robot working area 
using CAD software. Ting et al. [9] improved the accuracy and efficiency of robot, in the presence of obstacles and developed an algorithm for path planning of robots, used in industries.

\section{DESIGN ANALYSIS}

\section{Describing Robot Workspace}

Workspace of robot is the set of points that can be reached by robot end-effectors. For application of robot in industries or in any work environment, the workspace is very important to analyse. Evaluation of robot workspace is important to optimise the design process and for this, three things should be taken into consideration such as, the maximum reach of the robot, position workspace volume and orientation workspace volume. The 6 axis industrial robot and its workspace are shown below, in figure 1 and figure 2 , respectively.

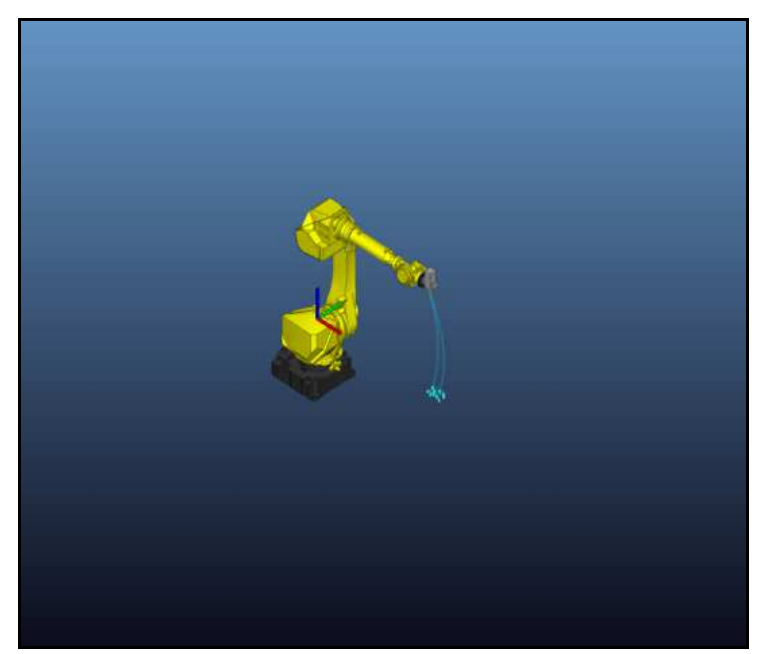

Figure 1: 6 Axis Industrial Robot

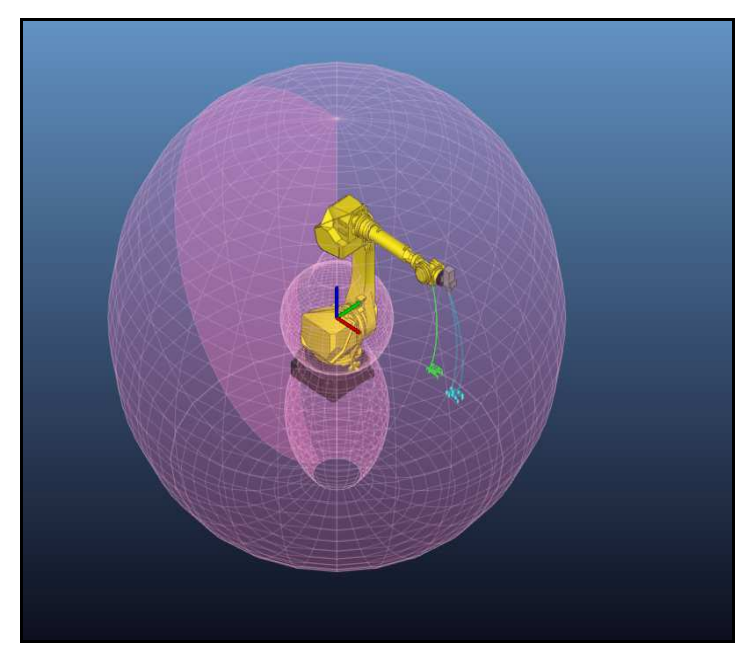

Figure 2: Workspace of Robot

\section{Path Planning of Industrial Robot}

For the design optimisation of robot, the layout of path has the major influence. The path with shortest path distance is considered as optimal path, as long as cycle time is concerned. Therefore, it is necessary to have idea about the paths followed by the robot and choose the path which can be useful, to optimise the design process. In this case, the optimisation criterion taken is the cycle time. For the analysis of cycle time taken by the industrial robot, ten numbers of 
tasks has been given, which is shown in below figure 2 and these are given the numbers, from 1 to 10 .

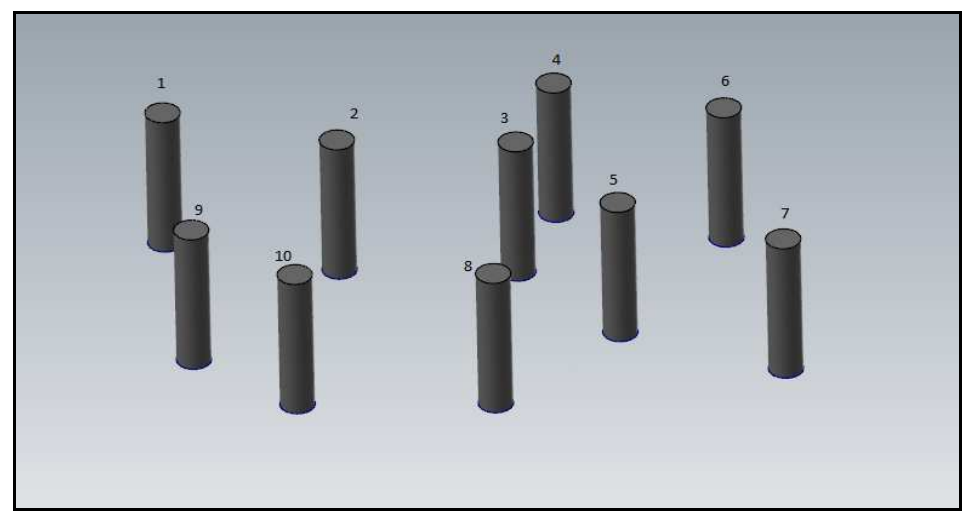

Figure 3: Arrangement of Tasks

For this particular analysis, a drilling tool is used which is replacing the griper of the industrial robot. The light blue line in the figure 3 shows the movement of the tool from home position, passing through all the tasks and returning back to its home position.

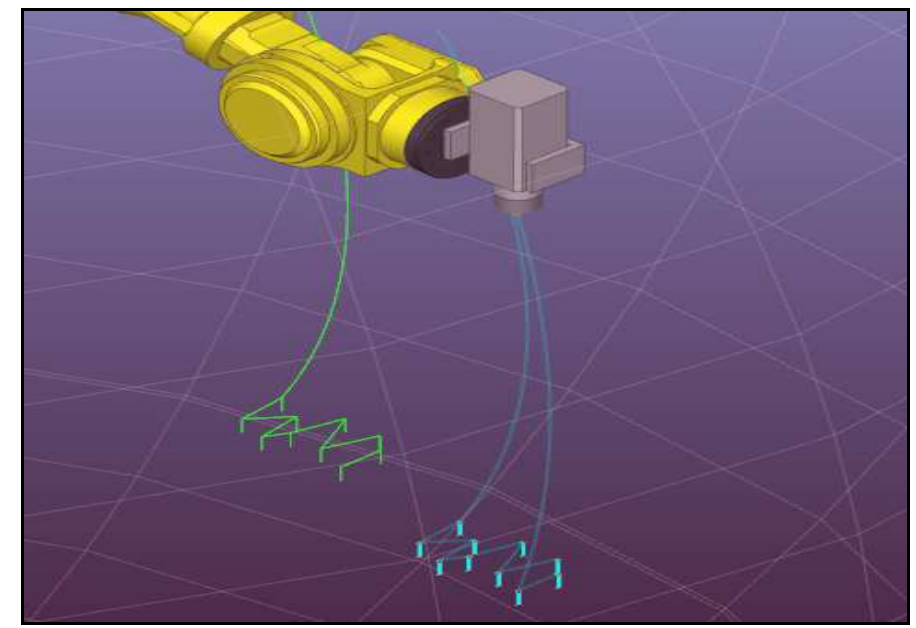

Figure 4: Robot Performance with in the Workspace

The six axis industrial robot consists of base, shoulder, elbow, wrist, pitch and roll. The base can revolve from 90 ${ }^{\circ}$ to $250^{\circ}$. It is the supporting lowest part of the robot. The shoulder can move between $-45^{\circ} \&-90^{\circ}$ for forward and backward motion of robot. During picking of the object, the elbow can play between $45^{\circ} \& 115^{\circ}$, for up and down movement. Wrist can play between $0^{\circ}$ to $340^{\circ}$. Pitch can move an angle between $-90^{\circ}$ to $90^{\circ}$ and roll can revolve between $0^{\circ}$ to $340^{\circ}$. Wrist, Pitch and roll, all make the robot flexible enough for picking and placing objects. Below Figure5, shows the movements of the six axises in $\mathrm{X}, \mathrm{Y} \& \mathrm{Z}$ coordinates. 


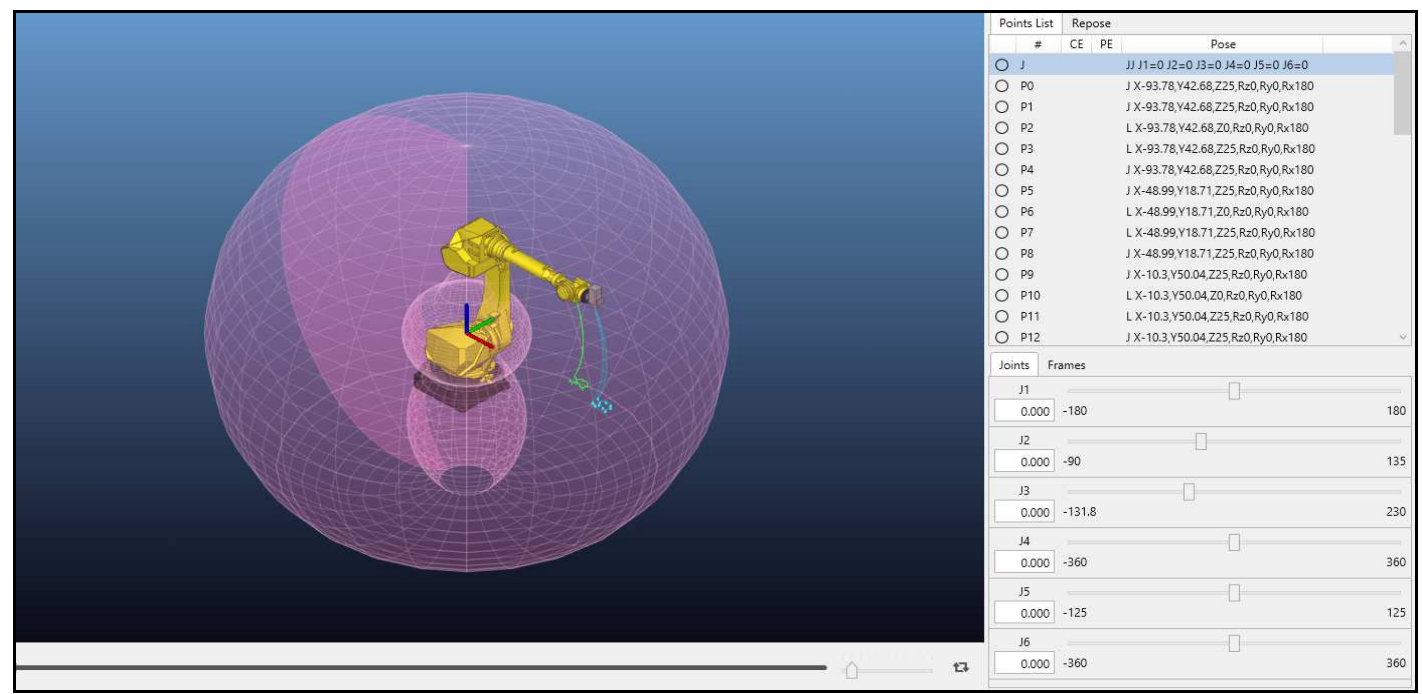

Figure 5: Movements of Six Joints in X, Y \& Z Coordinates

\section{RESULTS}

In order to analyze the performance of the robot, simulations were run several times, to obtain best value of cycle time. After analysing different paths followed by the robot to pass through ten tasks given, only seven paths have been selected, comparing with the path length and cycle time of each path. The Figure 5 shows the seven different paths followed by robot, to go through ten tasks. The left side figure shows the path and right side figure shows the cycle time, taken by the robot to follow that particular path, along with its path length. These figures are obtained from robomaster, by selecting different sort options for path planning of robot.

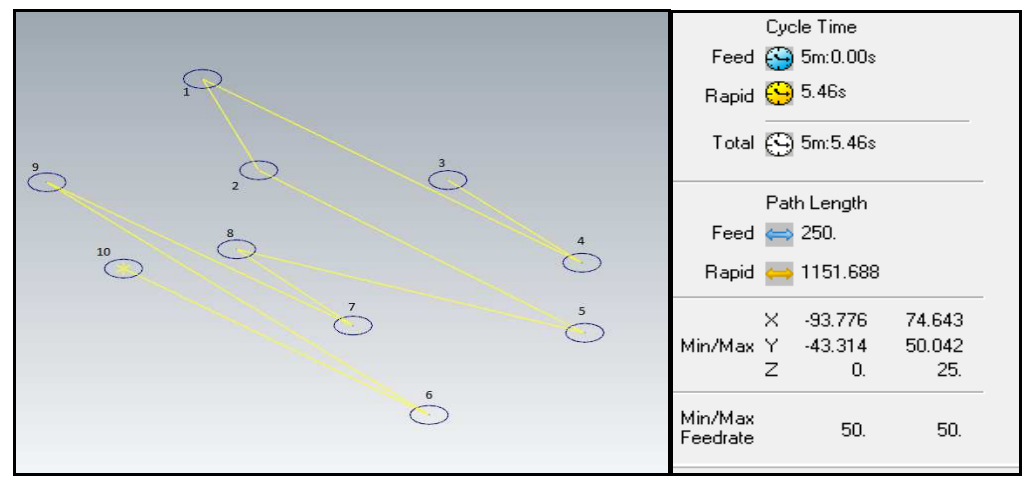

Path 1

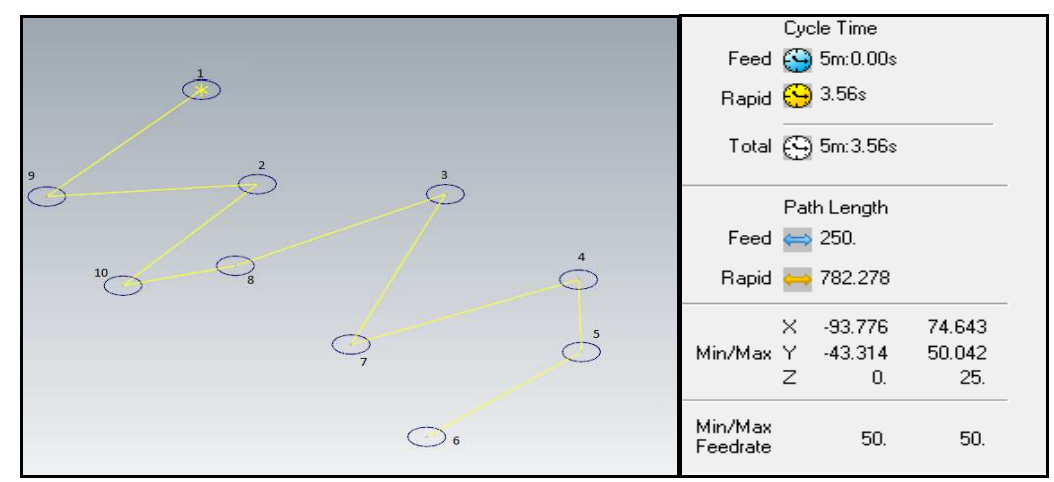

Path 2 


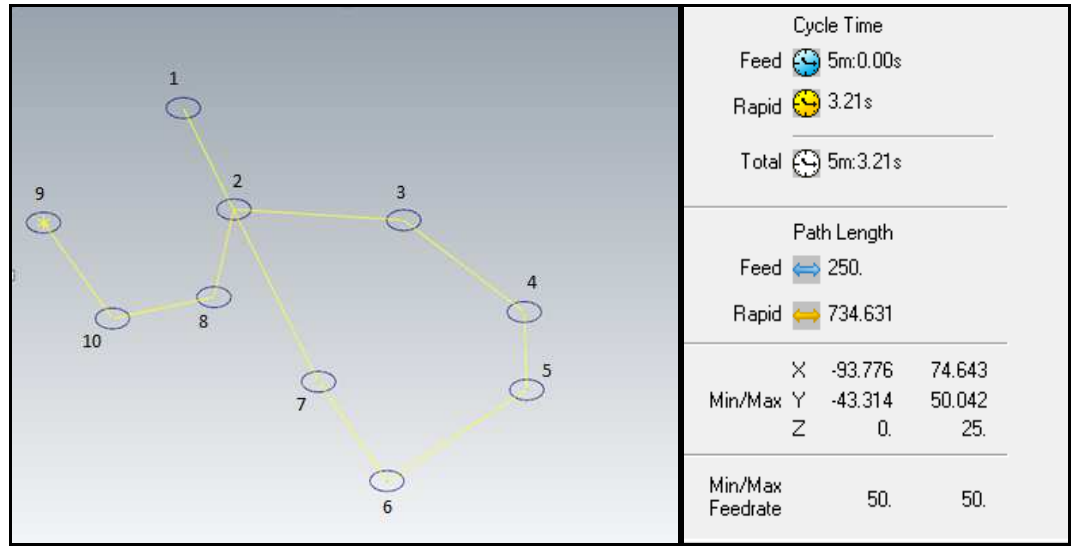

Path 3

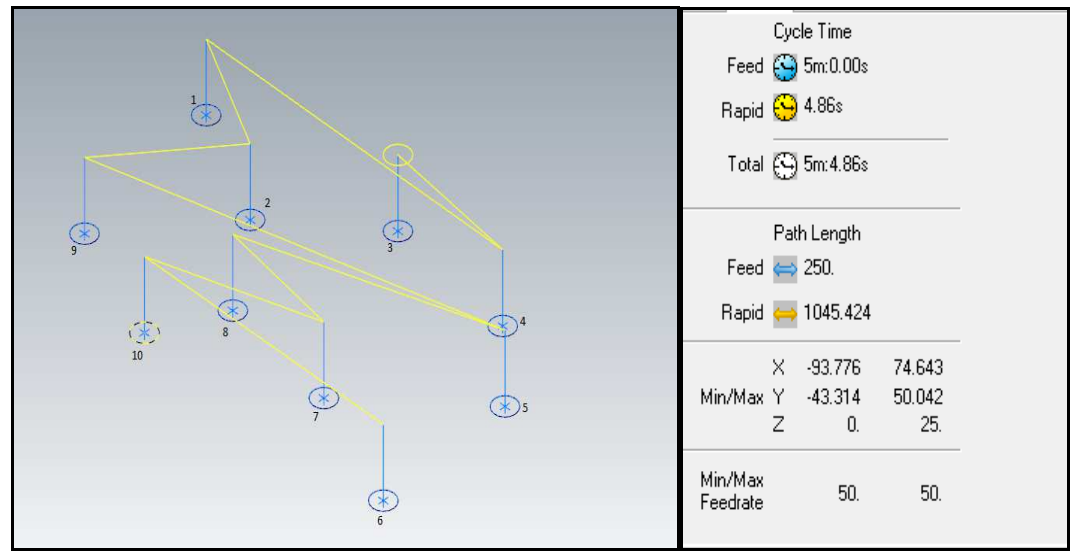

Path 4

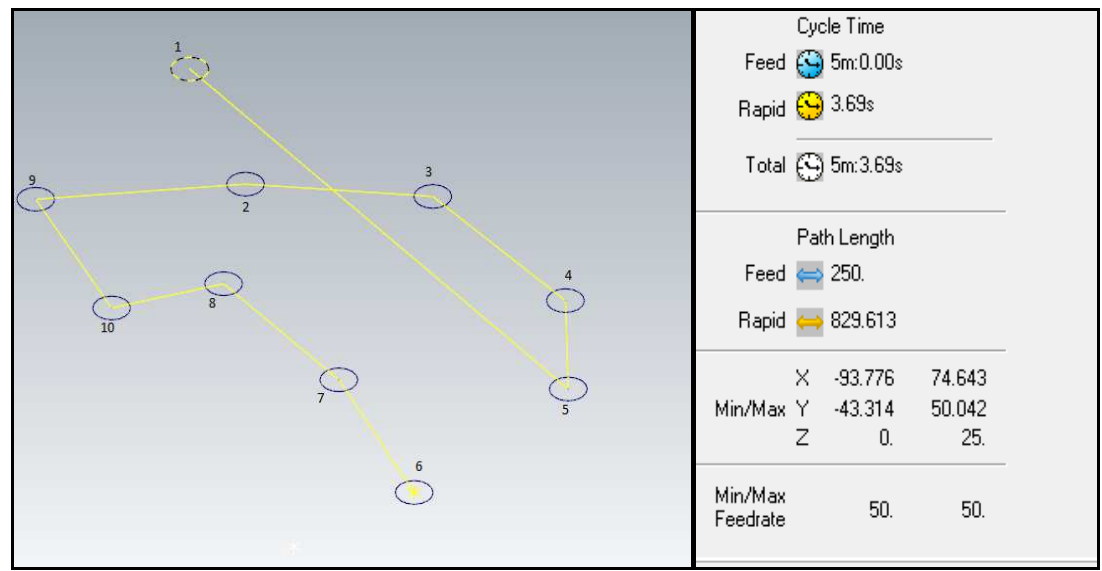

Path 5 


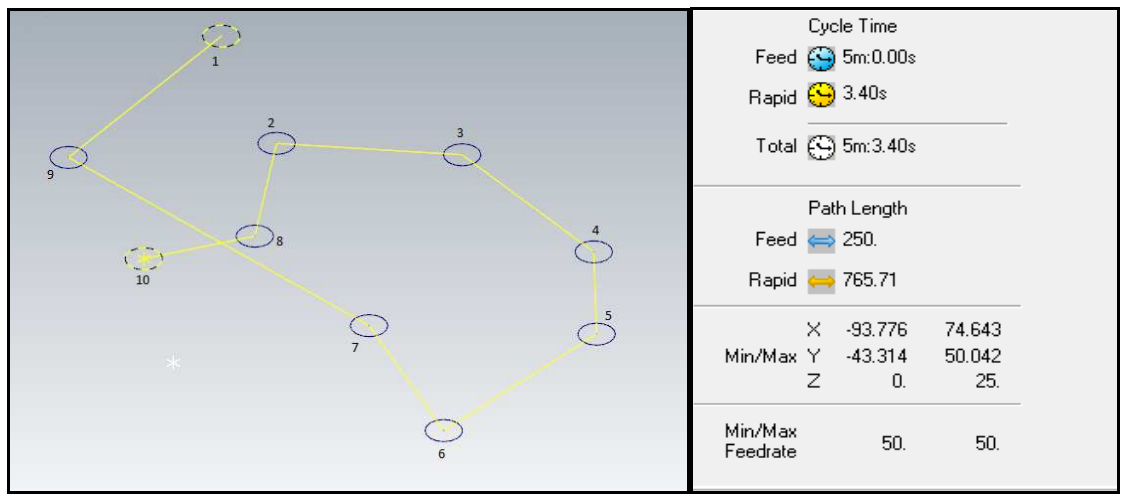

Path 6

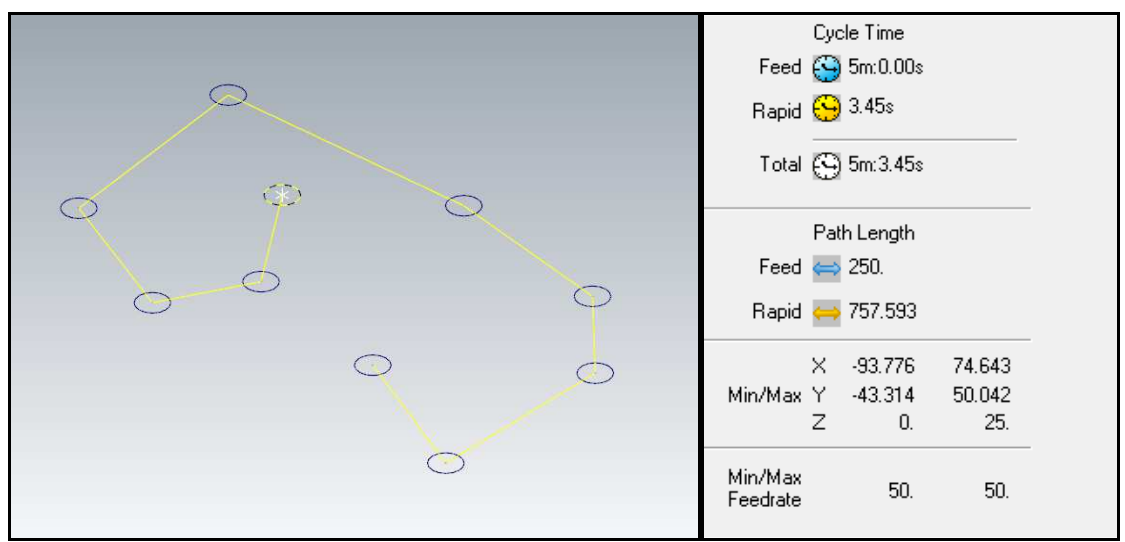

Path 7

Figure 5: Paths Followed by Robot for the Given Task

The Path Length and Cycle Time for seven different paths as discussed are presented in table 1. It is seen from the table that, for path number 1 the cycle time taken is 5.46 second, which is the highest value observed, corresponding to the path length of $1151.688 \mathrm{~mm}$. Whereas, for path number 3 the path length is $734.631 \mathrm{~mm}$, which is the lowest path length and cycle time taken is 3.21 second, which is the optimal cycle time period, taken by the robot to follow that path.

Table 1: Path Length and Cycle Time for Seven Different Paths

\begin{tabular}{|c|l|c|c|}
\hline S No & Paths & $\begin{array}{c}\text { Path Length } \\
\text { (mm) }\end{array}$ & $\begin{array}{c}\text { Cycle Time } \\
\text { (second) }\end{array}$ \\
\hline 1 & Path1 & 1151.688 & 5.46 \\
\hline 2 & Path2 & 782.278 & 3.56 \\
\hline 3 & Path3 & 734.631 & 3.21 \\
\hline 4 & Path4 & 1045.424 & 4.86 \\
\hline 5 & Path5 & 829.613 & 3.69 \\
\hline 6 & Path6 & 765.71 & 3.40 \\
\hline 7 & Path7 & 757.593 & 3.45 \\
\hline
\end{tabular}

The results from the simulation are presented below, in Figure 6 in the form of graphs. These two graphs (a and b) are nearly similar, because as path length increases, cycle time increases. It is seen from both the graphs that, for the path number 3 the lowest path length and the optimum cycle time is achieved. 


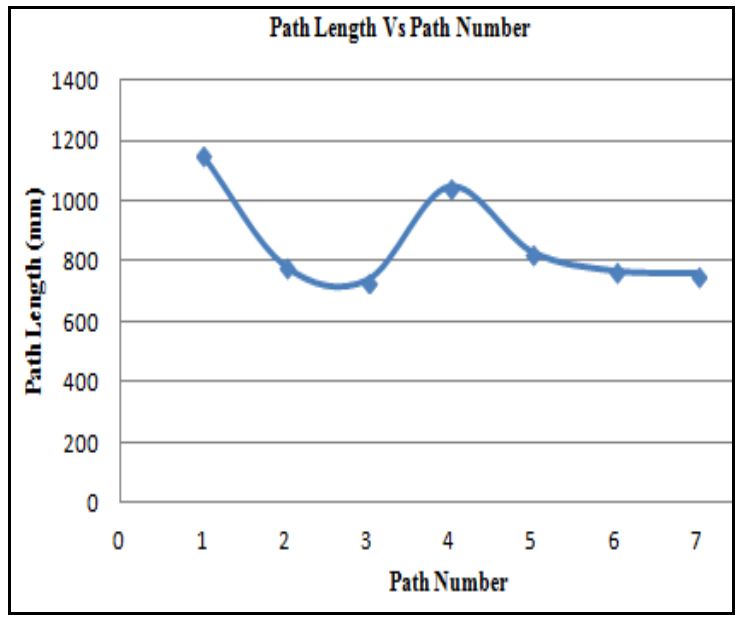

(a)

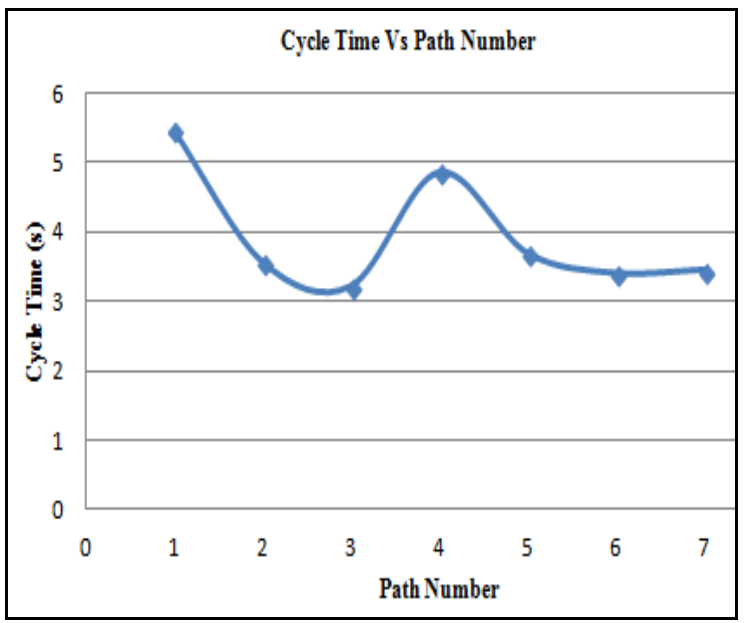

(b)

Figure 6: Comparison of Path Length (a) and Cycle Time (b) for Seven Different Paths

\section{CONCLUSIONS}

In this work, we evaluated and demonstrated the path planning method, in conjunction with shortest path traversal of the 6 axis industrial robot. Therefore, this paper proposes a solution for path planning, by obtaining the optimal cycle time with shortest path, followed using mastecam and robomaster software. Considering the workspace with its limit of working range, this method may be proposed for selection of tool path during machining with robot in industries or in any working environment. This proposed method may also be used for design optimisation of robots, to increase the flexibility and to make the robot more efficient and economic for future work.

\section{REFERENCES}

1. Carlson, J.S., Domenico Spensieria, C.,Warmfjord, K., Segeborn, J. (2014). Rikard Soderberg, Minimizing Dimensional Variation and Robot Traveling Time in Welding Stations. Procedia CIRP, 2377-82.

2. Ulricha, M., Lux, G., Jurgensen, L., Reinhart,G. (2016). Automated and Cycle time Optimized Path Planning for RobotBased Inspection Systems, procedia CIRP 44, 377-382.

3. Abele, E., Haehn, F., Pischan, M., Herr, F. (2016). Time optimal path planning for industrial robots using STL data files. Procedia CIRP 55, 6- 11. 
4. Yogita Gigras and Kusum Gupta. (2012). Ant Colony Based path planning Algorithm For Autonomous Robotic Vehicles. International journal of Intelligence and Applications (IJAIA), vol.3, No. 6.

5. Van Willigenburg, L.G., Hol, C.W.J., Van Henten, E.J. (2004). On-line near minimum-time path planning and control of an industrial robot for picking fruits, Computers and Electonics in Agriculture 44,223-237.

6. Cristinia Astejon, Giuseppe carbone, Garcia, J.C., Ceccarelli,M. (2010). A Multi-Objective Optimization of a Robotic Arm for Service Tasks. Journal of Mechanical Engineering 56, 316-329.

7. Saravanan, R., Ramabalan, S., Balamurugan, C., Subash, A. (2010). Evalutionary Trajectory Planning for an Industrial Robot, International Journal of Automation and Computing 7(2), 190-198.

8. Rahman, M. A. A., Osman, N. S., Boon, C. H. (2016). Configuring Safe Industrial Robot Work cell in Manufacturing Industry. Journal of Advanced Manufacturing Technology, Vol.10 No.2

9. Ting Y., Lei W.I., Jar H.C. (2002). Path planning algorithm for Industrial robots. Computers \& Industrial Engineering, Volume 42, Issues 2-4, 11 April, Pages 299-308. 